BMJ

Open

Gastroenterology

\title{
Point-of-care faecal calprotectin testing in patients with paediatric inflammatory bowel disease during the COVID-19 pandemic
}

\author{
Mwansa Jere (D) , ' Vikki Garrick, ${ }^{1}$ Lee Curtis, ${ }^{1}$ Rachel Tayler, ${ }^{1}$ Lisa Gervais, ${ }^{1}$ \\ Konstantinos Gerasimidis, ${ }^{2}$ Richard Hansen (D) ${ }^{1}$
}

To cite: Jere M, Garrick V, Curtis L, et al. Point-of-care faecal calprotectin testing in patients with paediatric inflammatory bowel disease during the COVID-19 pandemic. BMJ Open Gastro 2021;8:e000631. doi:10.1136/ bmjgast-2021-000631

- Additional supplemental material is published online only. To view, please visit the journal online (http://dx.doi. org/10.1136/bmjgast-2021000631).

Received 16 February 2021 Revised 9 April 2021 Accepted 11 April 2021
Check for updates

\section{(c) Author(s) (or their} employer(s)) 2021. Re-use permitted under CC BY-NC. No commercial re-use. See rights and permissions. Published by BMJ.

${ }^{1}$ Department of Paediatric Gastroenterology, Royal Hospital for Children, Glasgow, UK ${ }^{2}$ Department of Human Nutrition, University of Glasgow, Glasgow, UK

Correspondence to Richard Hansen; richard.hansen@glasgow.ac.uk

\section{ABSTRACT}

Objectives Following the disruption of normal paediatric inflammatory bowel disease (IBD) services during the peak of the COVID-19 pandemic, we prospectively audited the first-time use of home faecal calprotectin testing. We aimed to provide an alternative to laboratory tests and to assess the value of home testing as part of our regular services going forward.

Methods Home test kits as well as accompanying user instructions were made available to our patients with paediatric IBD that required faecal calprotectin test between 17 April and 12 August 2020. Once the user completed the test, results were automatically uploaded to the result portal and clinical staff were alerted. A user feedback questionnaire was sent to users that had completed the home test.

Results Of the 54 patients, 41 (76\%) aged between 4.7 and 18.1 years used the home test. A total of 45 home tests were done, one of which produced an invalid result. The decision to modify management was made in 12 (29\%) of the patients, while $14(34 \%)$ had no changes made and $15(37 \%)$ required further assessment. Twenty $(48.8 \%)$ responded to the questionnaire and $85 \%$ stated that they preferred the home test to the laboratory testing method.

Conclusions Home calprotectin tests were useful in guiding clinical management during a time when laboratory testing was less available. They may offer benefits as part of routine paediatric IBD monitoring to help target appointments and reduce unnecessary hospital attendances in the future.

\section{INTRODUCTION}

The emergence and global spread of the SARS-CoV-2 that causes the COVID-19 disease precipitated changes that disrupted paediatric inflammatory bowel disease (IBD) care, leading to abrupt service changes. ${ }^{1}$ During the first peak of the COVID-19 pandemic (April 2020), increased use of remote clinics and reduced face-to-face contact were recommended. Additionally, diagnostic procedures essential in the assessment of IBD including, endoscopy, histology, imaging and laboratory
Summary box

What is already known about this subject?

- Faecal calprotectin is a commonly used biomarker that provides a non-invasive method of monitoring bowel inflammation.

- Faecal calprotectin is most frequently quantitatively analysed in a laboratory setting using ELISA.

What are the new findings?

- Home faecal calprotectin using lateral flow assay is a relevant tool in maintaining contact-free monitoring of inflammatory bowel disease patients, of particular utility during the COVID-19 pandemic.

How might it impact on clinical practice in the foreseeable future?

- Home tests may allow continued objective monitoring of stable paediatric patients that may not require direct physical clinical assessment.

analysis for faecal calprotectin were no longer routinely available in many centres including our own. ${ }^{2}$ There was further concern about increased risk of severe COVID-19 infection in patients with IBD on immunosuppressant therapy, and several patients were advised to stay home and 'shield'. There was thus an immediate need to identify an interim method of monitoring patients and of identifying potential new cases of IBD while minimising unnecessary exposure to the virus for both patients and staff.

A survey of IBD services in the UK in April 2020 showed that most services did not have access to point-of-care calprotectin analysis. ${ }^{2}$ Within our service at Royal Hospital for Children, Glasgow (RHC), we initiated home faecal calprotectin testing for our patients with paediatric IBD to support their staying at home, to enhance virtual contact with objective data and to provide an objective 
but partial substitute for endoscopic assessment to guide treatment decisions.

We prospectively audited our use of home faecal calprotectin to explore the benefits of using home tests as part of the diagnosis and monitoring of disease activity in patients with paediatric IBD during the COVID-19 pandemic.

The IBDoc (Laboratories AG, Baselstrasse 55, CH-4124 Schönenbuch, Switzerland) home kit was selected owing to its wide usage in various countries, including some National Health Service (NHS) trusts, ${ }^{5-8}$ and as a kit which, when tested, was found to agree with ELISA tests at calprotectin levels $<500 \mu \mathrm{g} / \mathrm{g} .{ }^{9} 10$

\section{METHODS}

\section{Home calprotectin testing}

We included patients that were known to the paediatric gastroenterology team as well as potential new IBD cases. The patients were categorised into four groups: known patients reporting symptoms consistent with disease flare-up; known patients being monitored for response to current therapy; known patients undergoing routine surveillance of their disease activity; and suspected new IBD cases. The kits were either physically handed to the patients and families or mailed out in the post between 17 April and 12 August 2020.

IBDoc is a lateral flow immunochromatographic assay that can quantify calprotectin values between $30 \mu \mathrm{g} / \mathrm{g}$ and $1000 \mu \mathrm{g} / \mathrm{g} .{ }^{11}$ Values falling outside this range are recorded as $<30 \mu \mathrm{g} / \mathrm{g}$ or $>1000 \mu \mathrm{g} / \mathrm{g}$. The home calprotectin system relies on three categories of calprotectin results, with the defaults being $<100 \mu \mathrm{g} / \mathrm{g}$ normal, 100 $\mu \mathrm{g} / \mathrm{g}-300$ moderate and $>300$ high. $^{12}$ There is provision to adjust the categories, and we chose to categorise results of $0-250 \mu \mathrm{g} / \mathrm{g}$ as normal, $250-500 \mu \mathrm{g} / \mathrm{g}$ as moderate and values $>500 \mu \mathrm{g} / \mathrm{g}$ as high as these are the ranges commonly used in our clinical practice. ${ }^{13}{ }^{14} \mathrm{We}$ recognised the $<400 \mathrm{ug} / \mathrm{g}$ threshold from Walkiewicz et $a l^{4}$ as also supportive of remission but adjusted upwards to allow a spread of result. All results $>250 \mathrm{ug} / \mathrm{g}$ were considered as representing active disease and discussed by the clinical team, and decisions were made based on clinical correlation.

Families were provided a link to the company instruction video or given a run through the instructions by telephone or during a remote clinic. Our specialist IBD nurses set up accounts for patients once they had been given an IBDoc kit. Once the users felt they had understood the instructions, they were able to set up the IBDoc software on their smartphones and perform the test at home. The process involves installing a smartphone application (CalApp) that uses the phone camera to read the unique barcode and the result indicated on the test cassette. ${ }^{12}$ Initially, the user was able to see what category (high, medium or low) their result fell under; however, this was later reset for them to see only a blue screen and neither their actual calprotectin values nor the category.
This was done in order to reduce any anxiety that may have been caused by an unexpected result, particularly if the test was done out of hours when our team could not be contacted for advice.

\section{Laboratory calprotectin}

As laboratory processing for calprotectin was reintroduced, patients were able to submit samples. This included patients to whom kits had been posted, but for whom results had not yet been received, and there was urgency in obtaining a calprotectin level. Also included were patients who were advised to submit a sample to the lab following a home test as well as those for whom this was the more convenient option at the time. The lab calprotectin kits were collected either from the GP or the hospital (whichever was more practical), with some patients being able to produce a stool sample and submit the same day and others taking the kit home and handing the sample in as soon as they could. The laboratory uses ELISA to analyse faecal calprotectin and is able to quantify values between $30-1800 \mu \mathrm{g} / \mathrm{g}$ (values outside this range are reported as $<30 \mu \mathrm{g} / \mathrm{g}$ or $>1800 \mu \mathrm{g} / \mathrm{g}$ ).

\section{User feedback}

As this was the first time we had used home testing as part of our service, it was essential to obtain feedback from the patients and their families. All the families who had used IBDoc were sent a link to a short feedback survey using Webropol survey and feedback tool (online supplemental material). We designed a questionnaire that comprised of six closed-ended questions (two questions offered the option to enter free text) and two open-ended questions where the users were able to use their own words to explain why they preferred a particular testing method and how they felt about the home test. Our questionnaire was not formally validated; however, it was reviewed by our IBD team for face and content validity. Families received two email reminders to complete the survey.

As this study was a service evaluation, no ethical approval was required, and no hypothesis testing or sample size calculation was performed. Categorical variables were described as absolute frequencies and percentages, and the Mann-Whitney $\mathrm{U}$ test was used to compare median turnaround time by mode of home test kit delivery and laboratory processing. Data were analysed using IBM SPSS V.25.0 for Mac (IBM Corp, Armonk, New York, City, USA), and a $p$ value of $<0.05$ was regarded as statistically significant

\section{RESULTS}

IBDoc home test kits were made available to a total of 54 patients aged between 4.7 and 18.1 years (median age 15.3). Forty-four patients $(81.5 \%)$ received their home test kits via post, and the remaining $10(18.5 \%)$ patients were given theirs during face-to-face contact. Forty-five $(83.3 \%)$ were given to patients with Crohn's disease (one without full Porto assessment) ${ }^{15} 6$ (11.1\%) with ulcerative colitis (one without full Porto assessment) ${ }^{10}$ and 3 
Table 1 Patient characteristics

\begin{tabular}{lc}
\hline Total patients, n (\%) & $54(100)$ \\
\hline Male, $\mathrm{n}(\%)$ & $31(57.4)$ \\
\hline Female, $\mathrm{n}(\%)$ & $23(42.6)$ \\
\hline Crohn's disease, n (\%) & $45(83.3)^{\star}$ \\
\hline Ulcerative colitis, n (\%) & $6(11.1) \dagger$ \\
IBD type unclassified, n (\%) & $3(5.6)$ \\
Age at diagnosis <10, n (\%) & $21(39)$ \\
Age at diagnosis >10, n (\%) & $33(61)$ \\
\hline Immunosuppressive therapy, n (\%) & $47(87)$ \\
\hline
\end{tabular}

*Forty-four confirmed, one suspected but without full Porto assessment. $^{13}$

†Five confirmed, one suspected but without full Porto assessment. $n$, number of patients.

(5.6\%) with IBD type unclassified. Thirty-one $(57.4 \%)$ patients were male and $23(42.6 \%)$ were female. A total of $21(38.9 \%)$ were diagnosed with IBD under the age of 10 years. Forty-four $(81.5 \%)$ of the patients were on at least one immunosuppressant medication (table 1).

Ten $(18.5 \%)$ of the patients were given the home calprotectin kit after reporting symptoms consistent with flaring, 22 (40.7\%) for routine surveillance, 20 (37\%) for monitoring response to therapy and $2(3.7 \%)$ were new suspected cases of IBD. Of the 54 patients who received an IBDoc kit, $41(76 \%)$ used the test, while $13(24 \%)$ did not (table 2). A total of 45 home tests were completed on 41 patients. Results were reviewed on the IBDoc portal between 27 April and 22 August 2020.

The median calprotectin value was $509 \mu \mathrm{g} / \mathrm{g}$ (Q1: 197, Q3: 943) with 23 results (51\%) falling into the high range, $5(11 \%)$ moderate, $16(36 \%)$ normal and $1(2 \%)$ test was invalid. The single invalid test was as a result of user error, but the family was able to follow this with a valid repeat test. The management was modified in 13 (31.7\%) patients based on the IBDoc result and subsequent clinical correlation. This included dose adjustments, changes of medication and recommencement of medications. In
$15(36.7 \%)$ patients, the home test was used to establish that no changes were needed. It was decided that further assessment was required in $13(31.7 \%)$ of the patients. This included confirmatory laboratory faecal calprotectin, other lab tests and reassessment with endoscopy and MRI following discussion at multidisciplinary team meetings. One patient was advised to come in for a faceto-face clinical review, while medication compliance was discussed with another patient.

\section{User feedback}

Fourteen patients completed both a home and laboratory calprotectin test. These results are presented for interest but were not taken from the same stool sample or on the same day and so are not directly comparable (online supplemental material). When comparing result return time, however, lab calprotectin was comparable with home kit if given to families face to face (median 6 days vs 6.5 days, $\mathrm{p}=0.980$ ). Results were however significantly slower if the home kit was posted versus standard lab calprotectin (median 11 days vs 6 days, $\mathrm{p}=0.001$ ).

One challenge of home faecal calprotectin testing was the use of a separate and commercially provided electronic portal to access results that did not immediately or automatically feed into the patient record. Results therefore had to be manually transcribed to the electronic patient record. This increases the workload and introduces a potential opportunity for error while also making this a secondary source material for future casenote review for medicolegal challenge, etc.

Forty-one users were sent a link to a survey and 20 (48.8\%) responded by 31 August 2020. Fourteen (70\%) found the kit easy to use with the rest struggling to a certain degree (figure 1). Nineteen respondents (95\%) were willing to use the IBDoc test kit in the future with 17 $(85 \%)$ saying they preferred the home calprotectin kit to laboratory testing (figure 1). The most common reasons given for preferring the home testing methods included convenience (elimination of the need to travel long distances or make several trips) and quicker availability

Table 2 Summary of home kits received and completed tests by indication for faecal calprotectin

\begin{tabular}{llllll}
\hline & Flaring & Surveillance & $\begin{array}{l}\text { Response to } \\
\text { therapy }\end{array}$ & $\begin{array}{l}\text { New } \\
\text { Diagnosis }\end{array}$ & All \\
\hline IBDoc kit recipients, $\mathrm{n}(\%)$ & $10(100)$ & $22(100)$ & $20(100)$ & $2(100)$ & $54(100)$ \\
\hline Kits posted, $\mathrm{n}(\%)$ & $9(90)$ & $22(100)$ & $13(65)$ & $0(0)$ & $44(81)$ \\
\hline Kits face-to-face, $\mathrm{n}(\%)$ & $1(10)$ & $0(0)$ & $7(35)$ & $2(100)$ & $10(19)$ \\
\hline IBDoc result, $\mathrm{n}(\%)$ & $6(60)$ & $17(77)$ & $14(70)$ & $2(100)$ & $41(76)$ \\
\hline IBDoc result only, $\mathrm{n}(\%)$ & $4(40)$ & $14(64)$ & $8(40)$ & $0(0)$ & $26(48)$ \\
\hline Lab result only, $\mathrm{n}(\%)$ & $4(40)$ & $2(9)$ & $2(10)$ & $0(0)$ & $8(14.8)$ \\
\hline IBDoc + lab result, $\mathrm{n}(\%)$ & $3(40)$ & $3(14)$ & $6(30)$ & $2(100)$ & $14(26)$ \\
\hline No result, $\mathrm{n}(\%)$ & $0(0)$ & $3(14)$ & $4(20)$ & $0(0)$ & $6(11)$ \\
\hline Repeat IBDoc test & $1(10)$ & 0 & $2(10)$ & $1(50)$ & $4(7)^{*}$ \\
\hline
\end{tabular}

${ }^{*}$ One repeated for invalid result, three as part of continued monitoring.

$\mathrm{n}$, number of patients. 
1. Did you find the user instructions easy to understand?

Number of respondents (n) 20

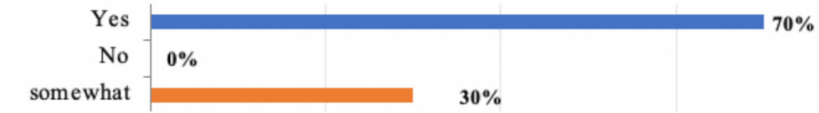

2. Did you find the IBDoc kit easy to use?

Number of respondents (n) 20

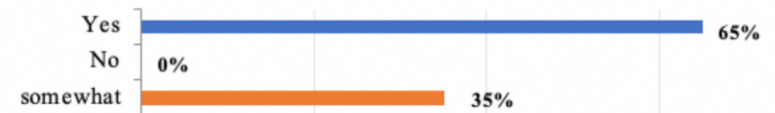

3. Is there anything you would add to the kit to make it easier to use? * Number of respondents (n) 20

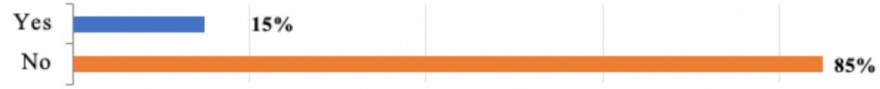

4. Would you be comfortable/willing to use the IBDoc home test kit in future? Number of respondents (n) 20

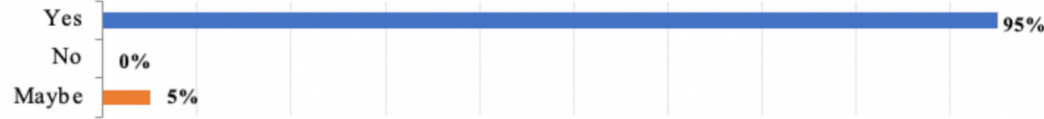

5. Do you have any suggestions on changes that could make the IBDoc easier to use? * Number of respondents (n) 19



6. Do you prefer the home sample kit or the hospital sample method? Number of respondents (n) 20

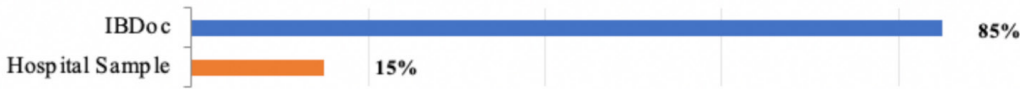

Figure 1 Survey results of user experiences with point-of-care calprotectin kits.

of results and ease of use of the home test. Additionally, patients felt that they were more comfortable and less anxious when doing the test at home (figure 2). Selected quotes from users include: 'my son gets very anxious at hospital so this kit is perfect to keep him happy'; 'my daughter prefers it as it's less messy, she's in control and

Reasons for preference of home kit over laboratory (total number respondents $n=16$ )

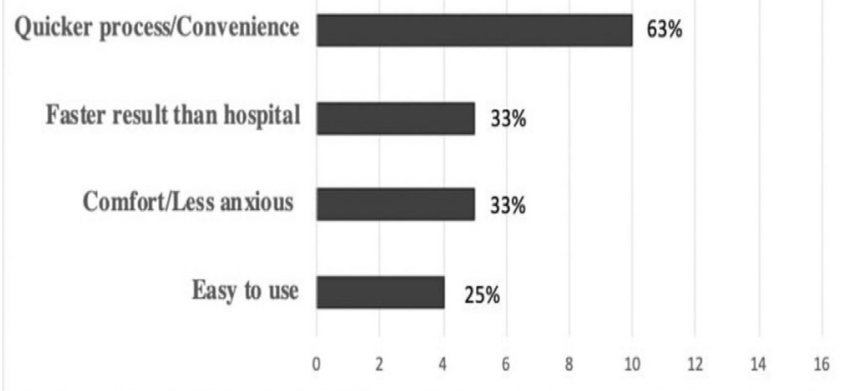

Figure 2 Reasons for preference of point-of-care calprotectin over laboratory alternative $(n=16)$. it's a more private experience'; and 'it was easy, I feel more comfortable'.

Users that preferred the laboratory method $(5 \%)$ all cited having more confidence in the accuracy of the hospital result as their reason. When asked to describe what users thought about the kit in their own words, the feedback was mostly positive. Several felt it was an interesting, practical and simple to use kit and others highlighted that it could be a bit awkward and tricky at the start (online supplemental material).

The cost of the home test kit was $£ 30$ plus postage, and the contract price of the laboratory calprotectin was $£ 30.73$. Neither of these amounts includes staff time and overhead costs.

\section{DISCUSSION}

Faecal calprotectin is a particularly sensitive biomarker for bowel inflammation and levels have been found to have a high correlation with endoscopic grading of IBD in both adults and children. ${ }^{16-18}$ With the disruption in services caused by the COVID-19 pandemic, the trialling of home calprotectin testing was essential in filling a 
gap in clinical service to our patients. Due to the project arising from the need to adjust to the COVID-19 associated hospital service restrictions that changed during the study period, particularly with regards to access to laboratory calprotectin, there was no predefined protocol. Consequently, there were no two tests (home and laboratory) conducted on the same stool sample to ascertain the correlation and agreement between the two methods. Indeed, this was not the purpose of the reported study. Studies from other centres have shown that although home tests do have higher variability, the calprotectin values were comparable with ELISA, particularly when calprotectin levels were $\leq 500 \mu \mathrm{g} / \mathrm{g}$. ${ }^{910}$

The majority of the test kits were posted out to patients and although the process was time consuming, particularly for our specialist IBD nurses, it allowed for faecal calprotectin results to be obtained by a contact-free method. This addressed some anxieties around COVID-19 that patients and their families were faced with, particularly in those who had been advised to shield as a result of the pandemic. However, owing to the lengthy mailing process, this mode may not have suited patients that were reporting a flare of their disease as there was more urgency in addressing their concerns and, importantly, home calprotectin appeared to be significantly slower in generating a result if sent by mail versus standard lab analysis in our study.

Home testing was also able to identify patients with high calprotectin who did not initially report symptoms prior to the test but later admitted to having symptoms or who were struggling with medication compliance. Additionally, in 28 (68\%) of the patients that did the home test, a decision was made about their clinical management based on the home test and clinical correlation alone without any further test. This demonstrates the potential usefulness of home testing during the COVID-19 pandemic and suggests this modality may be of continued utility as part of routine services for patients with IBD. Indeed, as physical outpatient attendance is likely to be restricted for some time to come and virtual consultations are now widespread, the additional objectivity of home faecal calprotectin testing could allow for triage/stratification of patient clinic attendance and targeting of review frequency and clinic type on a perpatient basis. Furthermore, there are many other areas where home testing might be a useful adjunct to clinical IBD services outwith the current COVID-19 pandemic, particularly where patients are geographically isolated, have transport difficulties or as a triage system for optimising clinic attendance in overburdened services. These potential opportunities warrant targeted investigation.

Feedback from the users that completed the survey was mostly positive and revealed that patients were less socially anxious and more comfortable using the home test kit as they felt they had control over both the environment and the timing. Lessening anxiety is an important reason to use home testing further, particularly in adolescents. Literature has shown this group to be significantly emotionally and psychologically affected by their disease activity, which is further compounded by their life stage and, for some, transition to adult care. ${ }^{19-21}$ It would be useful to examine how adolescent patients feel about home testing as this aspect was not adequately explored in this study as it was not one of the objectives. Additionally, further work is needed to explore the relatively high $(24 \%)$ rate of non-return/completion of home test kits we describe here if home calprotectin usage is to be increased and normalised in IBD practice. Of note, 8/13 $(62 \%)$ of the non-returning patients went on to complete a laboratory calprotectin, leaving five patients (9\%) where a calprotectin was requested with no contemporaneous result.

Although not a replacement for laboratory testing, home calprotectin testing was useful in guiding clinical decision making and may be worthy of adoption as part of routine IBD patient monitoring beyond the COVID-19 pandemic. It is important that a correlation is made between the test result and clinical symptoms, and possible additional investigations may be required for levels $>500 \mu \mathrm{g} / \mathrm{g}$ where the limited studies done have showed that results may be variable.

The initial cost of the two methods appear comparable; however, the home test minimises overheads and potentially reduces the patient costs and time involved in physically picking up/dropping off a sample kit. Cost as well as convenience for the patient are indeed to be considered in the process of implementing new IBD service pathways. It may be that a hybrid model involving laboratory calprotectin samples being mailed directly to the analysing laboratory could offer an alternative to home calprotectin, but this would require targeted consideration and significant infrastructure change. Going forward, there is a need to develop predefined guidelines to integrate home testing into routine clinical practice, alongside more conventional investigations, and to ascertain how the results of home testing can be made easily accessible in the patient's electronic medical records.

Contributors MJ contributed to questionnaire design, data collection, result analysis and preparation of manuscript; VG, LG and LC contributed to identification of participants, distribution of home tests, data collection, questionnaire design and review of manuscript; $\mathrm{RH}$ conceived the idea, gave clinical input, provided critical review of the manuscript; RT gave clinical input and manuscript review; KG provided scientific expertise and review of the manuscript.

Funding The paediatric inflammatory bowel disease team in Glasgow is supported by the Catherine McEwan Foundation. MJ's Clinical Research Fellowship is fully supported by the foundation. $\mathrm{RH}$ is funded by a National Health Service Research Scotland Career Researcher fellowship.

Competing interests None declared.

Patient consent for publication Not required.

Provenance and peer review Not commissioned; externally peer reviewed.

Data availability statement No data are available.

Supplemental material This content has been supplied by the author(s). It has not been vetted by BMJ Publishing Group Limited (BMJ) and may not have been peer-reviewed. Any opinions or recommendations discussed are solely those of the author(s) and are not endorsed by BMJ. BMJ disclaims all liability and responsibility arising from any reliance placed on the content. Where the content includes any translated material, BMJ does not warrant the accuracy and reliability 
of the translations (including but not limited to local regulations, clinical guidelines, terminology, drug names and drug dosages), and is not responsible for any error and/or omissions arising from translation and adaptation or otherwise.

Open access This is an open access article distributed in accordance with the Creative Commons Attribution Non Commercial (CC BY-NC 4.0) license, which permits others to distribute, remix, adapt, build upon this work non-commercially, and license their derivative works on different terms, provided the original work is properly cited, appropriate credit is given, any changes made indicated, and the use is non-commercial. See: http://creativecommons.org/licenses/by-nc/4.0/.

\section{ORCID iDs}

Mwansa Jere http://orcid.org/0000-0003-0497-3347

Richard Hansen http://orcid.org/0000-0002-3944-6646

\section{REFERENCES}

1 Ashton JJ, Kammermeier J, Spray C, et al. Impact of COVID-19 on diagnosis and management of paediatric inflammatory bowel disease during lockdown: a UK nationwide study. Arch Dis Child 2020;105:1186-91.

2 Kennedy NA, Hansen R, Younge L, et al. Organisational changes and challenges for inflammatory bowel disease services in the UK during the COVID-19 pandemic. Frontline Gastroenterol 2020;11:343-50.

3 Maclean A, Ashton JJ, Garrick V, et al. Impact of COVID-19 on the diagnosis, assessment and management of children with inflammatory bowel disease in the UK: implications for practice. BMJ Paediatr Open 2020;4:e000786.

4 Kennedy NA, Jones G-R, Lamb CA, et al. British Society of gastroenterology guidance for management of inflammatory bowel disease during the COVID-19 pandemic. Gut 2020;69:984-90.

5 Hejl J, Theede K, Møllgren B, et al. Point of care testing of fecal calprotectin as a substitute for routine laboratory analysis. Pract Lab Med 2018;10:10-14

6 Piekkala M, Alfthan H, Merras-Salmio L, et al. Fecal calprotectin test performed at home: a prospective study of pediatric patients with inflammatory bowel disease. J Pediatr Gastroenterol Nutr 2018;66:926-31.

7 Moore AC, Huang VW, Bourdages R, et al. IBDoc Canadian user performance evaluation. Inflamm Bowel Dis 2019;25:1107-14.

8 Parr H, Pavlidis P, Liew IY, et al. PTH-054 Home-Testing of Faecal Calprotectin Using the IBDoc ${ }^{\text {TM }}$ System: A Comparative Pilot Study. Gut 2016;65:A245.2-6.
9 Heida A, Knol M, Kobold AM, et al. Agreement between home-based measurement of stool calprotectin and ELISA results for monitoring inflammatory bowel disease activity. Clin Gastroenterol Hepatol 2017:15:1742-9.

10 Haisma S-M, Galaurchi A, Almahwzi S, et al. Head-To-Head comparison of three stool calprotectin tests for home use. PLoS One 2019;14:e0214751

11 Bühlmann Laboratories AG. IBdoc@ home test new patient support. Available: https://www.calprotectin.co.uk/covid-19/ibdoc-patientsupport/ [Accessed visited on 20/03/2021]

12 Bühlmann Laboratories AG. IBdoc®Instructions for use patients and lay users version 4.0, 2019. Available: https://www.alphalabs.co.uk/ media/productfile/file/i/f/ifu version 4.pdf

13 Henderson P, Casey A, Lawrence SJ, et al. The diagnostic accuracy of fecal calprotectin during the investigation of suspected pediatric inflammatory bowel disease. Am J Gastroenterol 2012;107:941-9.

14 Walkiewicz D, Werlin SL, Fish D, et al. Fecal calprotectin is useful in predicting disease relapse in pediatric inflammatory bowel disease. Inflamm Bowel Dis 2008;14:669-73.

15 Levine A, Koletzko S, Turner D, et al. ESPGHAN revised porto criteria for the diagnosis of inflammatory bowel disease in children and adolescents. J Pediatr Gastroenterol Nutr 2014;58:795-806.

16 Bunn SK, Bisset WM, Main MJ, et al. Fecal calprotectin as a measure of disease activity in childhood inflammatory bowel disease. J Pediatr Gastroenterol Nutr 2001;32:171-7.

17 Schoepfer AM, Beglinger C, Straumann A, et al. Fecal calprotectin correlates more closely with the simple endoscopic score for Crohn's disease (SES-CD) than CRP, blood leukocytes, and the CDAI. Am J Gastroenterol 2010;105:162-9.

18 Holtman GA, Lisman-van Leeuwen Y, Kollen BJ, et al. Diagnostic accuracy of fecal calprotectin for pediatric inflammatory bowel disease in primary care: a prospective cohort study. Ann Fam Med 2016;14:437-45

19 van den Brink G, Stapersma L, Vlug LE, et al. Clinical disease activity is associated with anxiety and depressive symptoms in adolescents and young adults with inflammatory bowel disease. Aliment Pharmacol Ther 2018;48:358-69.

20 Reigada LC, Hoogendoorn CJ, Walsh LC, et al. Anxiety symptoms and disease severity in children and adolescents with Crohn disease. $J$ Pediatr Gastroenterol Nutr 2015;60:30-5.

21 Patton GC, Sawyer SM, Santelli JS, et al. Our future: a Lancet commission on adolescent health and wellbeing. Lancet 2016;387:2423-78 